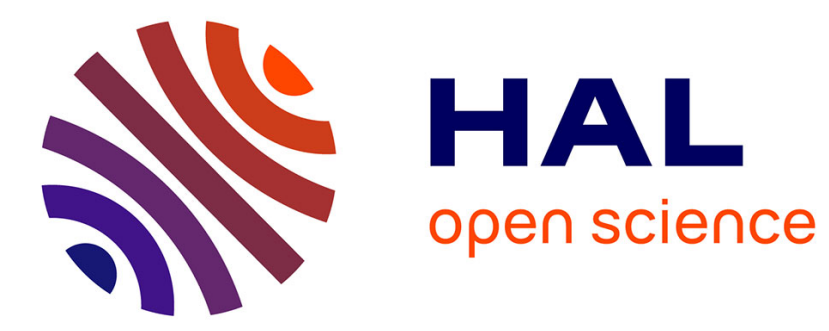

\title{
Nonlinear dynamic response analysis of damaged laminated composite structures
}

Saber Mahmoudi, Frédérique Trivaudey, Noureddine Bouhaddi

\section{To cite this version:}

Saber Mahmoudi, Frédérique Trivaudey, Noureddine Bouhaddi. Nonlinear dynamic response analysis of damaged laminated composite structures. Conference on Design and Modeling of Mechanical Systems, Mar 2015, Hammamet, Tunisia. hal-02963348

\section{HAL Id: hal-02963348 \\ https://hal.science/hal-02963348}

Submitted on 10 Oct 2020

HAL is a multi-disciplinary open access archive for the deposit and dissemination of scientific research documents, whether they are published or not. The documents may come from teaching and research institutions in France or abroad, or from public or private research centers.
L'archive ouverte pluridisciplinaire HAL, est destinée au dépôt et à la diffusion de documents scientifiques de niveau recherche, publiés ou non, émanant des établissements d'enseignement et de recherche français ou étrangers, des laboratoires publics ou privés. 


\title{
Nonlinear dynamic response analysis of damaged laminated composite structures
}

\author{
S. Mahmoudi ${ }^{1, a}$, F. Trivaudey ${ }^{2, a}$ and N. Bouhaddi ${ }^{3, a}$ \\ ${ }^{a}$ Applied Mechanics Department, FEMTO-ST Institute - UMR 6174, University of Franche-Comté-24, \\ chemin de l'Épitaphe, 25000 Besançon, France \\ ${ }^{1}$ saber.mahmoudi@femto-st.fr \\ ${ }^{2}$ frederique.trivaudey@univ-fcomte.fr \\ ${ }^{3}$ noureddine.bouhaddi@femto-st.fr
}

\begin{abstract}
In this work, a macro modeling is proposed to predict the dynamic response of damaged laminates made of unidirectional orthotropic layers of a polymer matrix reinforced with long fibers. The dynamic behavior of the composite structure is expressed through elasticity coupled with damage based on phenomenological approach for cracked structures. The structure is considered orthotropic but the damage is completely described by a single scalar variable whose evolution law is determined from the maximum dissipation principle. The incremental linear dynamic governing equations are obtained by using the classical KirchhoffLove theory of plates. Then, assuming that the damage induces nonlinearity, the obtained nonlinear dynamic equations are solved in time domain by Newmark method where an unconditionally stable scheme and iteration procedure are used. According to the numerical results, the mechanical behavior of the structure significantly change when the damage is taken into account. Under an impact load, damage increases and reaches its highest value with the maximum of the applied load and then remains unchanged. Besides, the eigenfrequencies of the damaged structure decrease comparing to the undamaged ones. This methodology can be used for monitoring strategies and life time estimations of hybrid complex structures because of the damage state is known in space and time.
\end{abstract}

Keywords: nonlinear dynamics, damage prediction, structural monitoring, laminated structures. 


\section{Introduction}

Composite materials are defined as the combination of more than two different materials having different mechanical properties. This combination is performed so that the resulting material has a better mechanical behavior from that of the individual components. Comparing to metallic materials, composite structures are characterized by a high resistance / weight ratio (Reddy 1997) which explains their wide use in the industry. Over the last decades, composite materials are increasingly used especially in high-tech products. As an example, the new project of aircraft such as Airbus A-380, where about $25 \%$ of the total weight of the aircraft is made of composite materials. They are characterized by sufficient strength with low density. In addition, they have other characteristics such as their resistance to corrosion and chemical attack. The composite structure is often subject to static or dynamic loads which can affect the mechanical properties and consequently which can change either the static or the dynamic responses (stress distribution and the deformation field or eigenfrequencies and mode shapes). These changes can induce a significant reduction in the structure lifetime. Various damage indicators are used to characterize the change in dynamic characteristics caused by the damage, such as natural frequencies (Vestroni and Capecchi 2000). In 1971, Kulkarni and Frederick (Kulkarni and Frederick 1971) have studied the presence and detection of delamination in a circular cylindrical composite shell through the decrease of eigenfrequencies. This idea was further investigated and deepened by Cawley and Adams (Cawley and Adams 1979). They have suggested that the shift of eigenfrequencies can be considered as a basis for a new nondestructive control technique. The change or the shift in the eigenfrequencies is then considered as an indicator able to report the damage evolution in composite structures. Thus, detection of the location and especially the degree of severity of the damage is of great importance in order to ensure the reliability and safety of service structures. Detecting damage using changes in the dynamic characteristics has been the subject of many research in the last years.

In this paper, a new investigation to predict the dynamic behavior and the damage evolution in composite structures, is proposed and developped. Based on a phenomenological model for cracked structures (Boubakar et al. 2002), the dynamic behavior is expressed through elasticity coupled with damage. The studied structures are made of unidirectional orthotropic layers of polymer matrix renforced with long fibers where the damage is expressed by a single scalar variable. The principal of maximum dissipation is used to determine the evolution of the damage which results in a material nonlinearity. Then, using the classical Kirchhoff-Love theory, the resulting nonlinear formulation is implemented in MATLAB ${ }^{\circledR}$ software. Then, a numerical solution is obtained based on a Newmark unconditionally stable algorithm with a prediction-correction scheme. Several numerical simulations have been performed showing 
that, under an impact load, damage increases and reaches its highest value with the maximum of the applied load and then remains unchanged. Besides, the eigenfrequencies of the damaged structure decrease compared with those of an undamaged one. Indeed, the displacement of the damaged structure increases due to the decrease in rigidity parameters.

\section{Problem formulation}

Initially used for modeling isotropic structures, the plate theories were later extended to model composite structures. A little reminder is provided, in this paragraph, around the formulations used to solve a dynamic problem in the case of composite structures. These formulations are essentially divided into two categories; those which are based on the Equivalent Single Layer Theory and those that are based on the Layer-Wise theory . The first category includes mainly the Classical Laminate Theory, the First-order (FSDT) and High-order (HSDT) Shear Deformation Theories, while the second category includes the Independent and Dependent layers theories. According to Reddy (Reddy 1997), it is recommended to use a theory from the first category if the global response of the stucture is unknown such as the eigenfrequencies and mode shapes in dynamic analysis. In the present work, The FSDT is retained for its simplicity of implementation and also because of the small thikness of the studied structures. So, the displacement field of a plate is written as follow:

$$
\{\underline{\mathbf{u}}(x, y, z, t)\}=\left\{\begin{array}{c}
u(x, y, z, t) \\
v(x, y, z, t) \\
w(x, y, z, t)
\end{array}\right\}+z\left\{\begin{array}{c}
\varphi_{y}(x, y, t) \\
-\varphi_{x}(x, y, t) \\
0
\end{array}\right\}
$$

Where $\varphi_{x}(x, y, t)$ and $\varphi_{y}(x, y, t)$ are the rotation, respectively, with respect to the $\vec{x}$ and $\vec{y}$ axes. The mechanical behavior is considered elastic. Noting that $E_{p}$ and $E_{c}$ are respectively the total potential and the kinetic energies of the structure, and according to virtual work principle, then:

$$
\delta \int_{t_{1}}^{t_{2}}\left(E_{p}+E_{c}\right) d t=0
$$

The problem is discretized by finite elements method using a quadrangular element Serendip $Q 8$ with eight nodes. The choice of this type of element is justified by its excellent performance in finite element modeling of thin and thick composite stuctures (CHEE 2000). Using the Gaussian integration method with 4 integration points where the integration weight is assumed equal to 1 , and a single point in the thickness direction located in the middle of each layer thickness, the global stiffness $[K]$ and mass $[M]$ matrices of the laminated structure consisting of " $\mathrm{n}$ " layers are obtained. In addition, the damping matrix is supposed proportional, and it is expressed by $[B]=\alpha_{1}[K]+\alpha_{2}[M]$. Finally, the dynamic equation of motion 
can be written as :

$$
[M] \ddot{u}+[B] \dot{u}+[K(D)] u=F
$$

The damage describes the creation of cavities and cracks within the structure (Lemaitre et al. 2001), that is in other words, the development of micro-discontinuities in the structure during the loading F. According to Boubakar et al (Boubakar et al. 2002), the effect of the damage on the elastic behavior depends on the micro-cracks opening mode as depicted in figue 1. Then, a three parameters $H_{22}, H_{44}$ and $H_{66}$ are introduced to characterise those
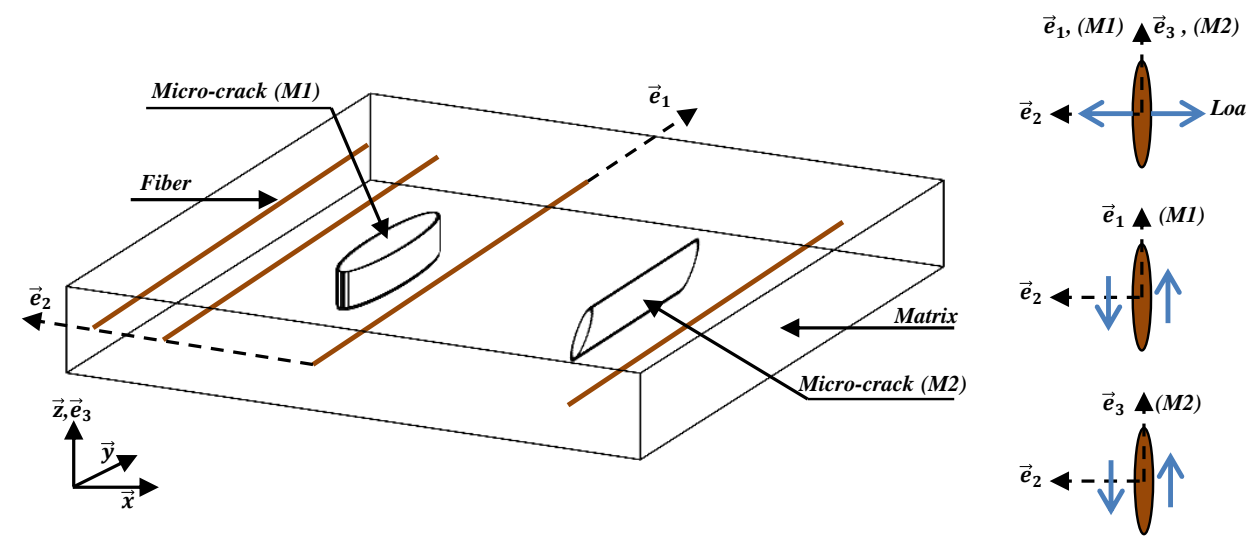

Figure 1: Micro-cracks orientation in the matrix (Boubakar et al. 2002)

effects respectively on the transverse Young's modulus $E_{2}$, shear modules $G_{12}$ and $G_{23}$. A self-consistent method (Perreux and Oytana 1993) permits to define a damage matrix $[H(D)]$ where the damage is fully expressed by a single scalar variable $\boldsymbol{D}$ which represents the relative reduction of the transverse Young's modulus:

$$
[H(D)]=\left[\begin{array}{cccccc}
0 & 0 & 0 & 0 & 0 & 0 \\
0 & H_{22} & 0 & 0 & 0 & 0 \\
0 & 0 & 0 & 0 & 0 & 0 \\
0 & 0 & 0 & H_{44} & 0 & 0 \\
0 & 0 & 0 & 0 & 0 & 0 \\
0 & 0 & 0 & 0 & 0 & H_{66}
\end{array}\right] \quad ; \quad\left\{\begin{array}{l}
H_{22}=\frac{D}{1-D} S_{22} \\
H_{44}=\frac{D}{\sqrt{1-D}} \sqrt{S_{11} S_{22}} \\
H_{66}=\frac{D}{\sqrt{1-D}} S_{22}
\end{array}\right.
$$

Where $S_{i i}$ depict the flexibility components of $[S]$ of the undamaged material. The damaged flexibility matrix $[\tilde{S}]$ is expressed in function of $[S]$ and the damage matrix $[H(D)]$ as follows:

$$
[\tilde{S}]=[S+H(D)]
$$

For an undamaged case $D=0,0<D<1$ for a damaged case, but there is a complete failure of the structure when $D=1$. The damage evolution law is expressed by using the 
thermodynamics of irreversible processes. A thermodynamic force $Y$ associated to the damage is obtained, $Y=\frac{1}{2} \sigma^{T}\left(\frac{\partial H}{\partial D}\right) \sigma$, where $\sigma^{T}$ is the transpose of the stress tensor $\sigma$ and $\left(\frac{\partial H}{\partial D}\right)$ is the derivative of $[H(D)]$ with respect to $D$. Indeed, a damage threshold function is defined as $\bar{Y}=Y_{c}+q D^{p}$ where $Y_{c}, q$ and $p$ are material constants. Thereby, the following charge function is established:

$$
f_{d}=Y-\bar{Y}=\frac{1}{2} \sigma^{T} \frac{\partial H}{\partial D} \sigma-\bar{Y}
$$

If $f_{d}<0$, the damage thermodynamic force $Y$ is less important than the damaged domain $\bar{Y}$, i.e. the damage does not yet appear. But, if the $f_{d}$ function tends to become positive, an increase of damage occurs so as to offset the increase of the function $Y$ and make $f_{d}$ and its derivative $\dot{f}_{d}$ null.

\section{Solving}

When the structure is subjected to a sufficient load able to trigger damage, this damaged state is kept in memory. So, it is recommended to use an incremental way in solving process. For each time iteration, a stress increment $\Delta \sigma$ is generated. This accumulation of stress continues until the function $f_{d}$ becomes positive. This implies the creation of a damage increment $\Delta D$ which will be obtained by solving the consistence equation $\dot{f}_{d}$. As a results, the solution is reduced to seek the damage increment $\Delta D$ which cancels the function $f_{d, i+1}$ for the $(i+1)^{t h}$ iteration, knowing the damage and stress states in $(i)^{t h}$ iteration, and writing the stress $\sigma_{i+1}$ us a function of $\Delta D$. So, the equation with the unknown variable $\Delta D$ is:

$$
f_{d, i+1}=\frac{1}{2}\left[\sigma_{i+1} H^{\prime}\left(D_{i}+\Delta D\right) \sigma_{i+1}\right]-\left[Y_{c}+q\left(D_{i}+\Delta D\right)^{p}\right]
$$

Where $H^{\prime}\left(D_{i}+\Delta D\right)$ is the derivative of the damage tensor $H$ with respect to $D$ and expressed at $\left(D_{i}+\Delta D\right)$. The equation (7) is solved using the Newton-Raphson method. After getting the damage increment, the stiffness matrix must be updated to take into account for this change of material properties. With this new stiffness, displacement, strain and stress are also updated and a residue is calculated to ensure the dynamic equilibrium expressed by the convergence criterion $\left\|\frac{r_{i+1}-r_{i}}{r_{i}}\right\|<\epsilon$.

The computing steps are detailed in the predictor-corrector alghorithm shown in Figure 2. For the step of damage test, in each Gauss point, a damage criterion is introduced such that when the damage $D_{i+1}$ exceeds 1 in a Gauss point, the stiffness in this point is assumed to be null. 


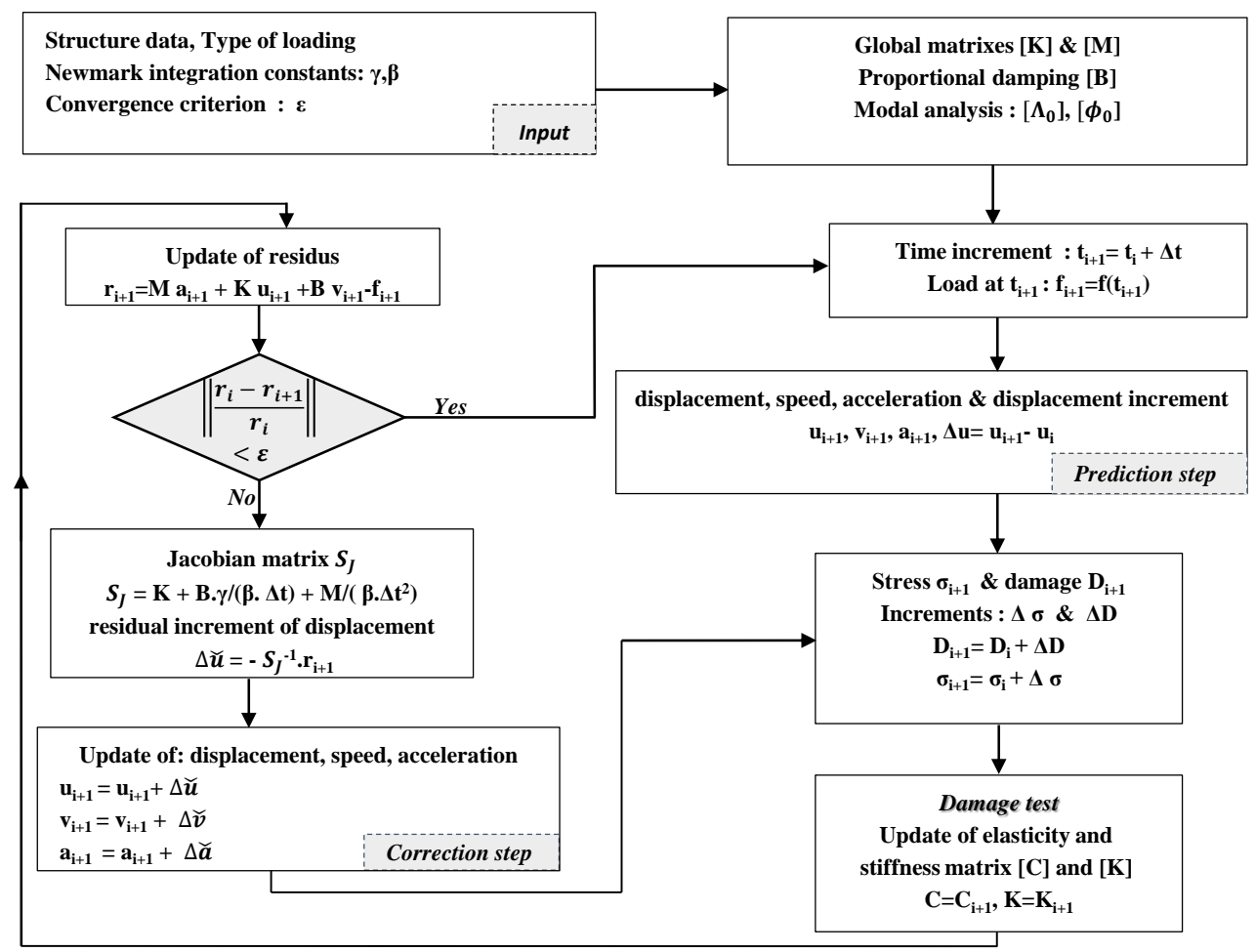

Figure 2: Dynamic predictor-corrector scheme for predicting the damage evolution and the nonlinear response of laminated damaged structure

\section{Results and discussion}

Several numerical simulations have been performed in order to highlight the mutual influence between damage and dynamic response. The geometrical and mechanical properties of the considered laminated beam are given in table 1 . The beam is made of three layers oriented as $\left(90^{\circ} / 0^{\circ} / 90^{\circ}\right)$, it is clamped at $x=0$ and free at $x=L$. It is subjected to a distributed impact along its free side and in $\vec{x}$ direction with $F=1971.5 \mathrm{~N}$ as magnitude and $\tau=1 \mathrm{~ms}$ as a duration.

In figure 3, the displacement amplitude is greater in the damaged case than in the undamaged one. Therefore, the damage has an important impact on the dynamic response since it affects the structure stiffness. This effect is visible in figure 4 and also when the eigenfrequencies of the undamaged and damaged beams are compared as in figure 5. So, when the damage is taken into account, the eigenfrequencies have a significant decrease which reaches up to $25 \%$ for the first mode. Moreover, comparing the damage evolution in curves (c) and (d) of the figure 3 , the damage decreases from the clamped side towards the free end of the beam. This can be explained by the fact that the tensile stress has also the same monotony. Also, 
Table 1: Geometrical and mechanical properties of the laminated beam

\begin{tabular}{ll}
\hline Elastic modulus $E_{1}\left(\vec{e}_{1}\right)$ & $45680 \mathrm{MPa}$ \\
Elastic modulus $E_{2}\left(\vec{e}_{2}\right)$ & $16470 \mathrm{MPa}$ \\
Shear modulus $G_{12}$ & $6760 \mathrm{MPa}$ \\
Poisson ratio $\nu_{12}$ & 0.34 \\
Poisson ratio $\nu_{23}$ & 0.34 \\
$Y_{c}$ & $0.0027 \mathrm{MPa}$ \\
$\mathrm{q}$ & $1.246 \mathrm{MPa}$ \\
$\mathrm{p}$ & 0.816 \\
Beam length $(\vec{x}): L$ & $0.3 \mathrm{~m}$ \\
Beam width $(\vec{y}): b$ & $0.03 \mathrm{~m}$ \\
Beam thickness $(\vec{z}): e$ & $0.001 \mathrm{~m}$ \\
\hline
\end{tabular}

numerical simulations show that the damage has an exponential shape and its slope tends to be more vertical when the damage becomes increasingly important. From 30\%, the scalar variable $D$ increases considerably, and the function $f_{d, i+1}$ in equation (7) will no longer have solution in the real space, which means that the complete damage is reached in this area of the structure.

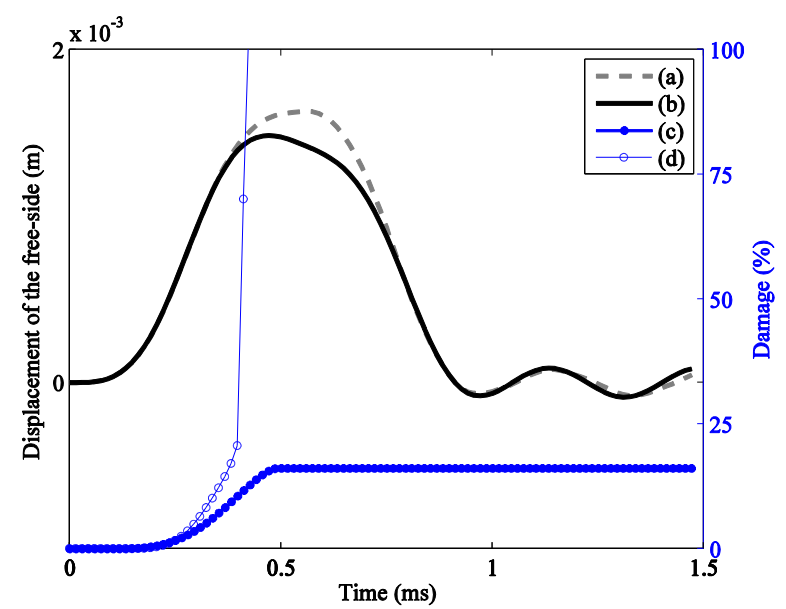

Figure 3: Dynamic impulse response; (a) damaged beam, (b) undamaged beam \& the damage evolution in time; (c) in a Gauss point located near the free side $(d)$ in a Gauss point located near the clamped side 


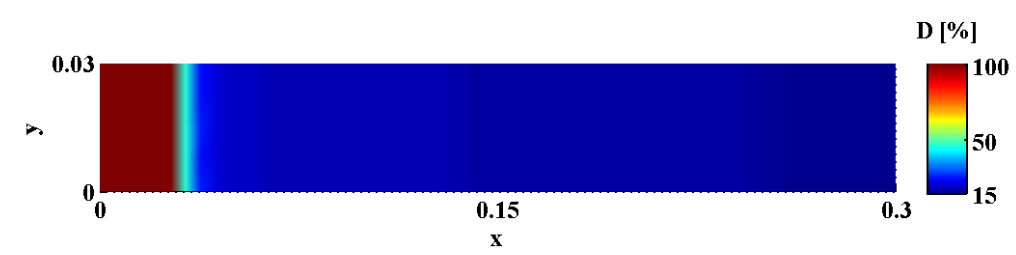

Figure 4: Damage propagation in the first layer oriented as $90^{\circ}$ of the laminated beam

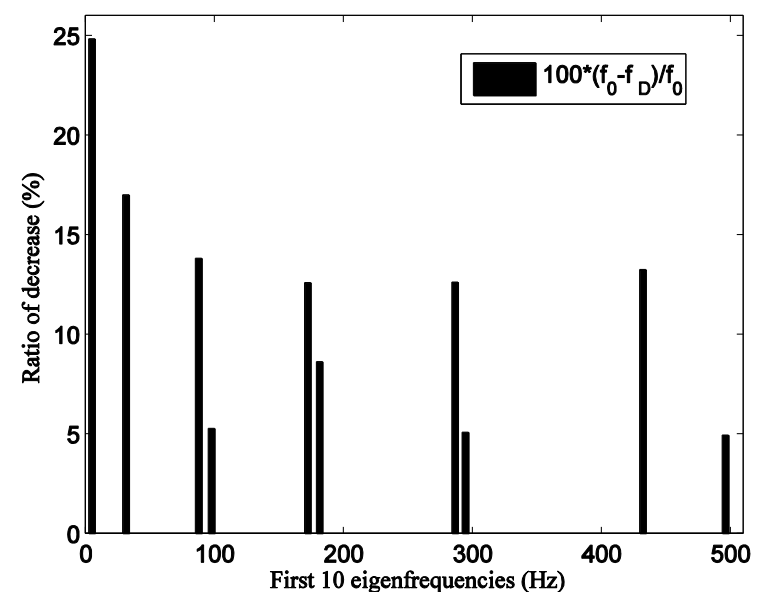

Figure 5: Decrease ratio between the eigenfrequencies, $f_{D} \& f_{0}$, respectively of damaged and undamaged laminated beam

\section{Conclusion}

The non-linear dynamic response of laminated beam was investigated including the material nonlinearity generated by damage. Hence, a macro modeling was proposed and investigated to predict the damage in the structure and its dynamic reponse under an impact load. Several numerical simulations have been performed to highlight the effect of the damage on the dynamic behavior, particularly, the eigenfrequencies and mode shapes. Also, the proposed modeling permits to predict the damage evolution in the space-time reference during loading. Hence, the damage modifies significantly the dynamic reponse reflected in the increase of the displacement response and the decrease between the eigenfrequencies of damaged and undamaged structures. This decrease can be reach up to $25 \%$ for the first natural frequency in the case of an impact load. Hence, the damage should not be neglected. The benefit of this investigation is damage predicting and its localizing in time and space. The changing effect of material properties induced by damage is taken account and introduced into the dynamic analysis during loading. Therefore, this methodology can be used in monitoring strategies and life time estimations of the complex structures. 


\section{References}

Boubakar M. L., Trivaudey F., Perreux D., Vang L. (2002) A meso-macro finite element modelling of laminate structures: Part i: time-independent behaviour. Composite Structures, pages 58:271-286.

Cawley P. and Adams R. D. (1979) A vibration technique for non-destructive testing of fiber composite structures. Journal of Composite Materials, 13:161-175.

CHEE C. Y. K. (2000) Static shape control of laminated composite plate smart structure using piezoelectric actuators. PhD thesis, University of Sydney, Aeronautical Engineering.

Kulkarni S. V. and Frederick D. (1971) Frequency as a parameter in delamination problems-a preliminary investigation. Journal of Composite Materials, 5:112-119.

Lemaitre J., Chaboche J. L., Benallal A., Desmorat R. (2001) Mécanique des matériaux solides. Dunod.

Perreux D. and Oytana C. (1993) Continuum damage mechanics for microcracked composites. Composites Engineering, 3:115-122.

Reddy J. N. (1997) Mechanics of laminated composites plate: Theory and Analysis. 2.ed. Florida: CRC Press.

Vestroni F. and Capecchi D. (2000) Damage detection in beam structures based on frequency measurements. Journal of Engineering Mechanics, 126:761-768. 